\title{
ABATACEPT AND LOW GAMMA-GLOBULIN LEVELS: NO ASSOCIATION WITH INFECTIOUS RISK OR RA DISEASE ACTIVITY CONTROL
}

Thiago Junqueira Trevisan ${ }^{1}$, Valquiria Garcia Dinis², Karina Rossi Bonfiglioli ${ }^{1}$, Andrea Yukie Shimabuco ${ }^{1}$, Carla Gonçalves Shahin Saad ${ }^{1}$, Julio Cesar Bertacini de Moraes ${ }^{1}$, Eloisa Silva Dutra de Oliveira Bonfá ${ }^{1}$, Ana Cristina de Medeiros Ribeiro ${ }^{1, \star}$

1.Universidade de São Paulo, São Paulo, Brazil. 2.Santa Casa de Misericórdia de Vitória, Vitória (ES), Brazil.

*Corresponding author: ana.medeiros@hc.fm.usp.br

\section{BACKGROUND}

Abatacept (ABA) can induce decrease in gamma-globulins, but the long-term safety of such reduction is unknown, as well its association with disease activity response. We evaluated ABA induced gamma-globulins reduction correlation with the risk of infections and the disease activity control in rheumatoid arthritis (RA) patients.

\section{METHODS}

This is a retrospective cohort of RA patients undergoing ABA in a single tertiary cohort (2007 to 2019). Patients were evaluated regarding clinical/inflammatory data, total and specific (IgG, IgM, IgA) gamma-globulins assessed before, at 3 and 6 months, and then every 6 months up to discontinuation/censoring. The occurrence of severe or recurrent infections were evaluated.

\section{RESULTS}

One hundred seventy-nine RA patients were included. They were predominantly female (93\%; $n=167)$, with positive rheumatoid factor (84\%; $n=151$ ). Median (range) age and disease duration: 55.1 (17-81.3) and 14 (1.6-39.8) years, respectively. Forty-five patients (25.1\%) had low IgG before ABA, but only 8 (4.5\%) had IgG 0.05. At baseline, TGG, IgG, IgM and IgA correlated positively to all disease activity parameters: DAS28 $(r=+0.33 ; r=+0.31 ; r=+0.24$ and $r=+0.37$, respectively; $p 0.05)$. Moreover, at 6 months, 25 patients (14\%) achieved DAS28 $<2.6$ and $49(27.4 \%)$ had low disease activity (DAS28 $<3.2$ ). Baseline or longitudinal measures of TGG and subtypes were similar among patients who responded and those who did not ( $p>0.05)$. Only 14 patients ( $9.4 \%$ ) had the medication discontinued due to infections: 3 recurrent infections and 11 severe infections. One patient died. The frequencies of low IgG and the levels of TGG and all subtypes at all time points were similar among patients with and without severe/recurrent infections $(p>0.05)$. None of these patients had low TGG or very low lgG $(<600 \mathrm{mg} / \mathrm{dL})$ at baseline or during treatment.

\section{CONCLUSION}

Reduction in gamma-globulin levels induced by ABA is safe and not associated to a higher risk of infection, even in patients with low IgG or TGG. Additionally, it is not associated to clinical response. 